\section{Kidney Blood Pressure Research}

\title{
The Association of Urinary Sodium and Potassium with Renal Uric Acid Excretion in Patients with Chronic Kidney Disease
}

\author{
Fengqin Li ${ }^{a, b}$ Hui Guo ${ }^{a, b}$ Jianan Zou ${ }^{a, b}$ Weijun Chen ${ }^{a, b}$ Yijun Lu ${ }^{a, b}$ \\ Xiaoli Zhang ${ }^{a, b}$ Chensheng Fu ${ }^{a, b}$ Jing Xiao ${ }^{a, b}$ Zhibin Ye ${ }^{a, b}$ \\ aDepartment of Nephrology, Huadong Hospital affiliated to Fudan University, Shanghai, bShanghai Key \\ Laboratory of Clinical Geriatric Medicine, Shanghai, China
}

\section{Key Words}

Chronic kidney disease - Urinary uric acid excretion - Urinary sodium excretion • Urinary potassium excretion

\begin{abstract}
Background/Aims: Hypertension and hyperuricemia are closely associated with an intermingled cause and effect relationship. Additionally, urinary sodium and potassium excretion is related to blood pressure. Whether or not it is associated with urinary uric acid excretion is not clear. Therefore, we aim to study the association of urinary sodium and potassium with renal uric acid excretion in patients with CKD. Methods: A cross-sectional study of 428 patients with CKD recruited from our department was conducted. All patients were divided into hypertension and non-hypertension group. In these two groups, Spearman correlation and multiple linear regression analysis were used to study the correlation of urinary sodium and potassium with renal handling of uric acid. Results: According to multiple linear regression analysis, in hypertension group, fractional excretion of sodium (FEna) was negatively correlated with 24 hour urinary uric acid (24-hUur) and uric acid clearance rate (Cur) (beta coefficients $[B]=-0.066,-0.182$, respectively; both $P<0.05$ ), and positively correlated with fractional excretion of uric acid (FEur) $(B=1.641, P<0.001)$. Additionally, fractional excretion of potassium (FEk) was positively correlated with FEur $(B=0.576, P<0.001)$, but not related to 24-hUur and Cur (both $P>0.05)$. And urinary sodium/potassium ratio (Una/k) was negatively related to $24-\mathrm{h}$ Uur and Cur $(B=-0.047,-0.159$, both $P<0.05)$, and positively related to FEur $(B=0.578, P<0.05)$. Furthermore, FEna and FEk was still positively related to FEur in the lowest tertile of eGFR groups (both $P<0.05$ ), but not related in the second and highest tertile of eGFR groups (all $P>0.05$ ). In non-hypertension group, FEna was negatively correlated with 24-hUur $(B=-0.589, P<0.05)$, but not related to Cur and FEur (both $P>0.05)$. both FEk and Una/k was not related to 24-h Uur, Cur and FEur (all $P>0.05$ ). Moreover, FEna and FEk was still not correlated

L. Fengqin and G. Hui contributed equally to this work.

\begin{tabular}{ll}
\hline Dr. Jing Xiao & Department of Nephrology, Huadong Hospital affiliated to Fudan University, \\
and Prof. Zhibin Ye & Shanghai 200040 (China) \\
& Tel. +8621-62483180-81008, Fax+8621-32140503, E-Mail jingxiao13@fudan.edu.cn, yezhibin3@126.com
\end{tabular}
\end{abstract}




\section{Kidney Blood Pressure Research}

with FEur in all tertiles of eGFR groups (all $P>0.05$ ). Conclusion: We found that in patients with $C K D$, urinary sodium and potassium excretion is closely correlated to renal handling of uric acid, which was pronounced in hypertensive patients with low eGFR. This phenomenon may be one of the mechanisms of the relationship between hypertension and hyperuricemia. Further research is needed to confirm it. It is expected to manage hyperuricemia in terms of controlling the diet of sodium and potassium.

\section{Introduction}

Hyperuricemia is common in chronic kidney disease (CKD) and associated with the development and progression of CKD [1-3]. The primary pathophysiology of hyperuricemia is defective renal handling of uric acid, including reduced glomerular filtration rate or enhanced reabsorption or insufficient secretion of uric acid by renal tubules [4, 5]. Proximal renal tubular epithelial cells (PTECs) express ion and urate transport channels responsible for reabsorption and secretion of urate [6]. Until now, the molecular mechanism underlying renal transport of uric acid and what factors may be involved in this process is not yet fully understood. Moreover, urinary uric acid excretion in population with CKD is rarely studied.

High sodium intake is a serious public health challenge in worldwide due to its contribution to the high prevalence and concomitant risks of hypertension and cardiovascular disease [7]. A study in 102, 216 adults from 18 countries showed a nonlinear association of sodium and potassium excretion with blood pressure [8]. Hypertension is a frequent finding in CKD, and most patients develop hypertension when the glomerular filtration rate (GFR) declines [9]. In addition, blood pressure of patients with CKD was more sensitive to high sodium intake than persons with normal kidney function due to a diminished capacity to excrete sodium [10]. On the other hand, hypertension and hyperuricemia are closely associated with an intermingled cause and effect relationship [11,12]. Early studies have reported that the reabsorption of uric acid and sodium by renal tubules are closely related, which may be associated with insulin resistance [13]. But clinical reports on the relationship between urinary sodium and uric acid excretion are few. Additionally, the relationship of urinary potassium with renal uric acid excretion is not clear. Therefore, in the present study, we analyzed the data from 428 adults with CKD divided into hypertension and nonhypertension groups to examine the association between urinary sodium and potassium excretion and renal handling of uric acid in a clinical setting. We look forward to a further understanding of the mechanisms of renal tubular transport of uric acid and the internal relationship between hypertension and hyperuricemia.

\section{Materials and Methods}

\section{Study participants}

In this cross-sectional study, 428 adult patients with CKD were admitted. Patients were excluded if they had taken drugs that could affect uric acid metabolism within the previous 2 weeks, such as diuretic, aspirin, losartan, cyclosporine, glucocorticoids, immunosuppressive agents, anti-tuberculosis drugs, sodium bicarbonate, levodopa, metformin, fenofibrate and UA-lowering agents, such as febuxostat, benzbromarone or allopurinol. Since these medicines CKD patients took could affect uric acid metabolism, thus interfering with the relationship between urinary sodium, potassium and uric acid, our protocol involved holding these agents for two weeks. Before collecting data, we carefully checked medical history of subjects in the electronic medical record system. If the subjects had stopped these medicines or changed to other drugs for at least two weeks, they had a chance to be included, which could make the effects of drugs on the results of the study minimized. All the patients were in a stable clinical condition. Those who had a history of kidney transplantation, dialysis, hereditary hyperuricemia, severe heart, lung or liver dysfunction, infection, tumor 


\section{Kidney Blood Pressure Research}

and hematologic diseases, hyperparathyroidism and shock were also excluded. Furthermore, all patients avoided high purine, fructose, sodium, and potassium diet and alcohol for 5 days before the study.

Our study was approved by the ethical review board, and it conformed to the ethics guidelines. Written informed consents were obtained from all subjects.

\section{Clinical and laboratory measurements}

A single $24 \mathrm{~h}$ urine sample was collected from each participant. All participants were instructed orally on the collection of $24 \mathrm{~h}$ urine samples. Each participant was provided with a three-liter plastic bottle. And they were asked to discard the first specimen in the morning, and from then on to collect all specimens for up to $24 \mathrm{~h}$. Trained nurses were in charge of recording the starting and ending time of each specimen collection and interviewing about completeness using a standard questionnaire. All samples were immediately sent to the clinical laboratory for the analysis of the 24-h urinary levels of uric acid (Uur), sodium (Una), potassium (Uk), urinary glucose (Ug), urinary volume (UV), urinary creatinine (Ucr), urinary albumin (UALB). Urinary albumin to creatinine ratio (UACR) was calculated as UALB/Ucr. Fractional excretion of uric acid (FEur) was calculated as (Uur $\times$ Scr $) /(S U A \times U c r) \times 100$, expressed as percentage. Uric acid clearance rate (Cur) was calculated as Uur $\times$ UV/SUA. Fractional excretion of sodium (FEna) was calculated as (Una $\times$ Scr)/ (Sna $\times$ Ucr $) \times 100$, expressed as percentage. Fractional excretion of potassium (FEk) was calculated as $(\mathrm{Uk} \times \mathrm{Scr}) /(\mathrm{Sk} \times \mathrm{Ucr}) \times 100$, expressed as percentage. Urinary sodium/potassium ratio was calculated as Una/ Uk. 24-h Uur, Cur and Ug were corrected for the body surface area.

Height, weight and blood pressure were measured by trained nurses in a standardized way. Body mass index (BMI) was calculated as weight in kilograms divided by height in meters squared. After 12 hours of fasting on the day of collecting 24 hours of urine, morning venous blood was sampled for routine biochemistry, including the serum levels of sodium (Sna), potassium (Sk), uric acid (SUA), creatinine (Scr), urinary nitrogen (BUN), C-reactive protein (CRP), total cholesterol (TC), triglycerides (TG), high-density lipoprotein (HDL), low-density lipoprotein (LDL), fasting blood glucose (FBG), total carbon dioxide (TCO2) and hemoglobin A1c (HbA1c). The estimated glomerular filtration rate (eGFR) (milliliters per minute per $1.73 \mathrm{~m}^{2}$ ), an indicator of renal function, was calculated using the Chronic Kidney Disease Epidemiology Collaboration (CKD-EPI) formula [14].

\section{Diagnostic and grouping criteria}

Hyperuricemia was defined as the serum level of uric acid $>420 \mu \mathrm{mol} / \mathrm{L}$; Hypertension was defined as systolic blood pressure (SBP) $\geq 140 \mathrm{mmHg}$, diastolic blood pressure (DBP) $\geq 90 \mathrm{mmHg}$, or use of antihypertensive medication according to self-report or to pharmacy data. Study participants were divided into hypertension group $(\mathrm{H})$ and non-hypertension group $(\mathrm{NH})$. In hypertension population, according to tertiles of eGFR, patients were divided into three groups (HG1: eGFR $<33.9 \mathrm{ml} / \mathrm{min} / 1.73 \mathrm{~m}^{2}$; HG2: $33.9 \leq \mathrm{eGFR}<66.6 \mathrm{ml} / \mathrm{min} / 1.73 \mathrm{~m}^{2}$; HG3: eGFR $\geq 66.6 \mathrm{ml} / \mathrm{min} / 1.73 \mathrm{~m}^{2}$ ). In non-hypertension group, according to tertiles of eGFR, patients were divided into three groups (NHG1: eGFR<68.4ml/min $/ 1.73 \mathrm{~m}^{2}$; NHG2: $68.4 \leq \mathrm{eGFR}<98.5 \mathrm{ml} / \mathrm{min} / 1.73 \mathrm{~m}^{2}$; NHG3: eGFR $\geq 98.5 \mathrm{ml} / \mathrm{min} / 1.73 \mathrm{~m}^{2}$ ). Renal handling of sodium and potassium were estimated with FEna, FEk and Una/k, which are less affected by the diet. The indexes of renal uric acid excretion include 24-h Uur, FEur and Cur. According to tertiles of 24-h Uur, all patients were

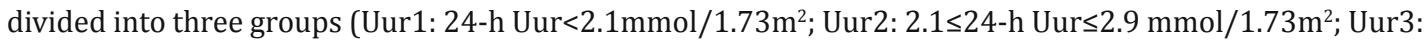
24-h Uur $>2.9 \mathrm{mmol} / 1.73 \mathrm{~m}^{2}$ ). In hypertension group, according to tertiles of FEna, patients were divided into three groups (FEna1: FEna<0.92\%; FEna2: 0.92\% 5 FEna $\leq 1.88 \%$; FEna3: FEna $>1.88 \%$ ). According to tertiles of FEk, patients were divided into three groups (FEk1: FEk<8.36\%; FEk2: 8.36\% $\leq$ FEk $\leq 15.17 \%$; FEk3: FEk>15.17\%); According to tertiles of Una/k, patients were divided into three groups (Una/k1: Una/

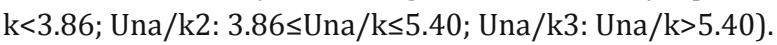

\section{Statistical analysis}

The continuous variables are reported in means \pm SD and categorical variables are presented in percentages. In case of nonparametric data distribution medians with inter quartile range (IQR) are presented. Comparisons were made between groups using Student's t-test or one-way ANOVA. We used least significance difference (LSD) test if the variance was homogeneous, and we used Tamhane's T2 test if not. Non-normally distributed data was compared using Kruskall-Wallis test. Categorical data was compared 


\section{Kidney Blood Pressure Research}

using the Chi-square test. Correlations were Pearson's or Spearman's depending on the distribution of the data. In the present study, TG, CRP, Ug, UACR, FEna, FEk, Una/k and FEur were non-normally distributed, thus, Spearman's correlation was used among these variables. If Spearman's correlation analysis was statistically significant, multiple linear regression analysis was performed. Before conducting the multiple correlation analysis, we firstly verified that independent variables were not correlated among them. We analyzed the multiple collinearity by calculating the correlation coefficient matrix, tolerance and variance inflation factor of independent variables. The result of analysis suggested that there was no multiple collinearity. Multiple linear regression analyses were then performed to determine the association of urinary sodium and potassium (FEna, FEk and Una/k) with renal handling of uric acid (24-h Uur, Cur and FEur) in hypertension and non-hypertension group. And we further analyzed the association of FEna and FEk with FEur in different groups stratified by tertiles of eGFR in hypertension and non-hypertension population, respectively. Statistical significance for all analyses was set at $P<0.05$. Statistical analysis was performed with software SPSS 22.0.

\section{Results}

Characteristics of the study population and when stratified by tertiles of 24- $h$ Uur

We included 428 participants with mean age 55.5 \pm 16.7 years and 219 males (51.2\%). There were 254 hypertensive patients. General data are shown in Table 1. Biochemical differences in different groups according to 24-h Uur tertiles are also summarized in Table 1. Participants in the highest tertile of 24-hUur group had lower age, SUA, Sk, proportion of hypertension, Una/k, FEna, FEk and FEur, higher eGFR, UV and Cur than those in the lowest tertile of 24-h Uur group (all $P<0.05$, Table 1 ).

Table 1. Characteristics of the study population according to tertiles of 24 -h Uur. ${ }^{*} \mathrm{P}<0.05,{ }^{* *} \mathrm{P}<0.01$ vs. the Uur1 using the least significance difference (LSD) method if the variance is equal or the Tamhane's T2 method if the variance is not equal, using Kruskal-Wallis test in case of nonparametric data distribution. Numbers are mean \pm SD or proportion. For TG, CRP, 24-hUg, FEna, FEk, Una/k, Cur, and FEur, median and the 25th and 75th percentile are shown. Abbreviations: BMI, body mass index; eGFR, estimated glomerular filtration rate; CRP, C-reactive protein; TC, total cholesterol; TG, triglycerides; HDL, high-density lipoprotein; LDL, low-density lipoprotein; FBG, fasting blood glucose; SUA, serum uric acid; Sna, serum sodium; Sk, serum potassium; 24-hUg, 24-hour urinary glucose; 24-h Uur, 24-hour urinary uric acid excretion; FEna, fractional excretion of sodium; FEk, fractional excretion of potassium; Una/k, urinary sodium/potassium ratio; Cur, uric acid clearance rate; FEur, fractional excretion of uric acid

\begin{tabular}{|c|c|c|c|c|c|}
\hline Variables & All $(n=428)$ & $\begin{array}{c}\text { Uur1 } \\
<2.13 \mathrm{mmol} / 24 \mathrm{~h} \\
\end{array}$ & $\begin{array}{c}\text { Uur2 } \\
2.13-2.89 \mathrm{mmol} / 24 \mathrm{~h}\end{array}$ & $\begin{array}{c}\text { Uur3 } \\
>2.89 \mathrm{mmol} / 24 \mathrm{~h}\end{array}$ & $P$ value \\
\hline Males gender(\%) & 51.2 & 45.4 & 53.7 & 54 & 0.259 \\
\hline Age(years) & $55.5 \pm 16.7$ & $60.5 \pm 16.0$ & $55.7 \pm 16.8^{*}$ & $47.9 \pm 15.8^{* *}$ & $<0.001$ \\
\hline $\operatorname{BMI}\left(\mathrm{kg} / \mathrm{m}^{2}\right)$ & $24.2 \pm 4.0$ & $23.2 \pm 3.9$ & $24.4 \pm 3.5^{*}$ & $24.3 \pm 3.7$ & 0.061 \\
\hline eGFR(ml/min/1.73 $\left.\mathrm{m}^{2}\right)$ & $64.4 \pm 36.4$ & $42.7 \pm 37.0$ & $67.2 \pm 33.8^{* *}$ & $86.8 \pm 28.8^{* *}$ & $<0.001$ \\
\hline SUA(umol/L) & $388.7 \pm 109.7$ & $403.3 \pm 127.3$ & $359.5 \pm 98.3^{* *}$ & $328.2 \pm 91.8^{* *}$ & $<0.001$ \\
\hline $\mathrm{TC}(\mathrm{mmol} / \mathrm{L})$ & $4.9 \pm 1.4$ & $4.7 \pm 1.1$ & $4.6 \pm 1.0$ & $4.9 \pm 1.5$ & 0.226 \\
\hline $\mathrm{TG}(\mathrm{mmol} / \mathrm{L})$ & $1.5(1.0,2.4)$ & $1.5(1.0,2.1)$ & $1.4(1.0,2.3)$ & $1 \cdot 5(1 \cdot 0,2.2)$ & 0.436 \\
\hline $\mathrm{HDL}(\mathrm{mmol} / \mathrm{L})$ & $1.3 \pm 0.4$ & $1.3 \pm 0.4$ & $1.3 \pm 0.4$ & $1.3 \pm 0.4$ & 0.789 \\
\hline $\mathrm{LDL}(\mathrm{mmol} / \mathrm{L})$ & $2.8 \pm 1.0$ & $2.8 \pm 0.9$ & $2.8 \pm 0.9$ & $2.9 \pm 1.2$ & 0.268 \\
\hline $\mathrm{FBG}(\mathrm{mmol} / \mathrm{L})$ & $5.1 \pm 1.6$ & $5.1 \pm 1.6$ & $5.3 \pm 1.9$ & $5.4 \pm 1.8$ & 0.375 \\
\hline $\mathrm{CRP}(\mathrm{mg} / \mathrm{L})$ & $2.7(0.8,6.2)$ & $2.4(0.8,5.5)$ & $2.7(0.8,6.3)$ & $3.0(0.8,6.3)$ & 0.907 \\
\hline $\mathrm{Sna}(\mathrm{mmol} / \mathrm{L})$ & $141.9 \pm 3.0$ & $142.3 \pm 2.5$ & $141.9 \pm 3.0$ & $141.7 \pm 3.6$ & 0.315 \\
\hline $\mathrm{Sk}(\mathrm{mmol} / \mathrm{L})$ & $4.0 \pm 0.4$ & $4.1 \pm 0.5$ & $4.0 \pm 0.5^{*}$ & $3.9 \pm 0.4^{* *}$ & $<0.001$ \\
\hline 24-hUV(mL) & $1849.2 \pm 662.1$ & $1650.5 \pm 609.7$ & $1882.2 \pm 600.3^{*}$ & $1960.0 \pm 791.7^{* *}$ & $<0.001$ \\
\hline 24-hUg(mmol/1.73 $\left.\mathrm{m}^{2}\right)$ & $0.6(0.3,2.3)$ & $0.6(0.2,2.9)$ & $0.5(0.3,2.1)$ & $0.7(0.4,1.8)$ & 0.783 \\
\hline Hypertension(\%) & 59.3 & 72.3 & 59.2 & 46 & $<0.001$ \\
\hline Diabetes(\%) & 28.0 & 26.2 & 33.3 & 23.7 & 0.170 \\
\hline FEna(\%) & $1.0(0.6,1.9)$ & $1.9(1.0,5.1)$ & $1.0(0.6,1.6)^{* *}$ & $0.7(0.5,0.9)^{* *}$ & $<0.001$ \\
\hline FEk(\%) & $8.9(6.2,14.5)$ & $14.9(8.3,28.9)$ & $8.5(5.6,13.0)^{* *}$ & $6.6(5.1,8.3)^{* *}$ & $<0.001$ \\
\hline $\mathrm{Una} / \mathrm{k}$ & $4.3(3.1,5.7)$ & $4.7(3.4,6.9)$ & $4.3(3.2,5.4)^{*}$ & $4.1(3.0,5.6)$ & 0.018 \\
\hline $\operatorname{Cur}\left(\mathrm{ml} / \mathrm{min} / 1.73 \mathrm{~m}^{2}\right)$ & $5.3(3.2,6.4)$ & $3.3 \pm 3.0$ & $5.3 \pm 1.8^{* *}$ & $9.1 \pm 5.4^{* *}$ & $<0.001$ \\
\hline FEur(\%) & $6.7(5.2,9.5)$ & $8.9(6.0,16.4)$ & $7.2(5.4,11.1)$ & $7.3(5.8,10.0)$ & 0.149 \\
\hline
\end{tabular}




\section{Kidney Blood Pressure Research}

Among the etiology of CKD, chronic glomerulonephritis (CGN) accounted for the highest proportion (71.3\%, 305/428), followed by hypertensive nephropathy (HN) (13.1\%, 56/428), and diabetic kidney disease (DKD) accounted for (11.7\%, 50/428). In addition, polycystic kidney disease (PKD) and atherosclerotic renal arterial stenosis (ARAS) accounted for $8.6 \%$ $(18 / 428)$ and 3.3\% (7/428). Among this population of CKD patients included in the study, calcium channel blockers, $\beta$ blockers, statins, $\alpha$-glucosidase inhibitor or insulin were used, and these agents were similar among groups.

Association of urinary sodium and potassium excretion with renal uric acid excretion

In hypertensive group, Spearman correlation analysis showed that FEna was negatively associated with 24-h Uur and Cur ( $\mathrm{r}=-0.436, P<0.001 ; \mathrm{r}=-0.528, P<0.001$, respectively), and positively associated with FEur ( $\mathrm{r}=0.696, P<0.001)$. FEk was negatively associated with 24 -h Uur and Cur ( $\mathrm{r}=-0.469, P<0.001 ; \mathrm{r}=-0.566, P<0.001$, respectively), and positively associated with FEur $(\mathrm{r}=0.645, P<0.001)$. Una/k was negatively related to 24 -hUur and Cur $(\mathrm{r}=-0.174$, $P<0.05 ; \mathrm{r}=-0.175, P<0.05)$, and positively related to FEur $(\mathrm{r}=0.330, P<0.001)$. Multiple linear regression analysis showed that the association of FEna and Una/k with 24-h Uur, Cur and FEur was still statistically significant (all $P<0.05$; Table 2, 3 and 4), FEk was still positively related to FEur $(P<0.001$; Table 4$)$, and the correlation with 24-h Uur and Cur disappeared $(P>0.05$; Table 2 and 3 ), after adjusting confounding factors in three models (model 1 adjusted for sex, age and BMI; model 2 plus eGFR and SUA; model 3 plus FBG, 24-hUg, TG, CRP, LDL, and TCO2).

In non-hypertensive group, Spearman correlation analysis showed that FEna was negatively associated with 24-h Uur and Cur ( $\mathrm{r}=-0.297, P<0.001 ; \mathrm{r}=-0.295, P<0.001$, respectively), and positively associated with FEur ( $\mathrm{r}=0.574, P<0.001)$. FEk was negatively associated with 24-h Uur and Cur ( $\mathrm{r}=-0.323, P<0.001 ; \mathrm{r}=-0.367, P<0.001$, respectively), and positively associated with FEur $(\mathrm{r}=0.467, P<0.001)$. Una/k was not related to 24 -hUur and $\operatorname{Cur}(\mathrm{r}=-0.062, P=0.415 ; \mathrm{r}=-0.016, P=0.165)$, and positively related to FEur $(\mathrm{r}=0.270, P<0.001)$. Multiple linear regression analysis showed that FEna was still negatively associated with 24-h Uur $(P<0.05$; Table 2), and the association of FEna with Cur and FEur disappeared (both $P>0.05$; Table 3 and 4). Moreover, the association of FEk and Una/k with 24-h Uur, Cur and FEur all disappeared (all $P>0.05$; Table 2, 3, and 4), after adjusting confounding factors in three models (model 1 adjusted for sex, age and BMI; model 2 plus eGFR and SUA; model 3 plus FBG, 24-hUg, TG, CRP, LDL, and TCO2).

In order to investigate how the association of FEna and FEk with FEur tracks with changes in eGFR, their relationship in three groups stratified by tertile of eGFR in hypertension and non-hypertension population was further analyzed as follows.

Table 2. Multiple linear regression analysis for the association of FEna, FEk, and Una/k (independent variable) with 24-hUur (dependent variable) in hypertension and non-hypertension group. model 1 adjusted for sex, age and BMI; model 2 plus eGFR and SUA; model 3 plus FBG, 24-hUg, TG, CRP, LDL, and TCO2. Beta coefficients refer to how many deviations a dependent variable will change per deviation increase in the predictor variable. SE refers to standard error. H: hypertension group; NH: non-hypertension group. Abbreviations: 24-h Uur, 24-hour urinary uric acid excretion; FEna, fractional excretion of sodium; FEk, fractional excretion of potassium; Una/k: urinary sodium/potassium ratio

\begin{tabular}{|c|c|c|c|c|c|c|c|c|c|c|}
\hline \multirow{2}{*}{ Parameter } & & \multicolumn{3}{|c|}{ Model 1} & \multicolumn{3}{|c|}{ Model 2} & \multicolumn{3}{|c|}{ Model 3} \\
\hline & & B & SE & $\mathrm{P}$ & B & SE & $\mathrm{P}$ & B & SE & $\mathrm{P}$ \\
\hline \multirow{2}{*}{ FEna (\%) } & $\mathrm{H}$ & -0.094 & 0.022 & $<0.001$ & -0.051 & 0.025 & 0.042 & -0.066 & 0.021 & 0.002 \\
\hline & $\mathrm{NH}$ & -0.299 & 0.121 & 0.014 & -0.329 & 0.030 & 0.028 & -0.589 & 0.256 & 0.024 \\
\hline \multirow{2}{*}{ FEk (\%) } & $\mathrm{H}$ & -0.020 & 0.005 & $<0.001$ & -0.009 & 0.006 & 0.168 & -0.012 & 0.006 & 0.058 \\
\hline & $\mathrm{NH}$ & -0.057 & 0.023 & 0.015 & -0.066 & 0.030 & 0.058 & -0.092 & 0.053 & 0.087 \\
\hline \multirow{2}{*}{ Una/k } & $\mathrm{H}$ & -0.057 & 0.021 & 0.007 & -0.037 & 0.020 & 0.065 & -0.047 & 0.020 & 0.021 \\
\hline & $\mathrm{NH}$ & -0.085 & 0.065 & 0.195 & -0.079 & 0.067 & 0.240 & -0.141 & 0.102 & 0.172 \\
\hline
\end{tabular}




\section{Kidney Blood Pressure Research}

Table 3. Multiple linear regression analysis for the association of FEna, FEk, and Una/k (independent variable) with Cur (dependent variable) in hypertension and non-hypertension group. model 1 adjusted for sex, age and BMI; model 2 plus eGFR and SUA; model 3 plus FBG, 24-hUg, TG, CRP, LDL, and TCO2. Beta coefficients refer to how many deviations a dependent variable will change per deviation increase in the predictor variable. SE refers to standard error. H: hypertension group; NH: non-hypertension group. Abbreviations: Cur, uric acid clearance rate; FEna, fractional excretion of sodium; FEk, fractional excretion of potassium; Una/k: urinary sodium/potassium ratio

\begin{tabular}{lcccccccccc}
\hline \multirow{2}{*}{ Parameter } & & \multicolumn{3}{c}{ Model 1 } & \multicolumn{3}{c}{ Model 2 } & \multicolumn{3}{c}{ Model 3 } \\
& & $\mathrm{B}$ & $\mathrm{SE}$ & $\mathrm{P}$ & $\mathrm{B}$ & $\mathrm{SE}$ & $\mathrm{P}$ & $\mathrm{B}$ & SE & $\mathrm{P}$ \\
\hline \multirow{2}{*}{ FEna (\%) } & $\mathrm{H}$ & -0.273 & 0.056 & $<0.001$ & -0.121 & 0.055 & 0.029 & -0.182 & 0.074 & 0.015 \\
& $\mathrm{NH}$ & -0.729 & 0.268 & 0.007 & -0.442 & 0.300 & 0.144 & -0.937 & 0.505 & 0.067 \\
\multirow{2}{*}{ FEk (\%) } & $\mathrm{H}$ & -0.068 & 0.013 & $<0.001$ & -0.017 & 0.014 & 0.220 & -0.027 & 0.023 & 0.248 \\
& $\mathrm{NH}$ & -0.168 & 0.050 & 0.001 & -0.132 & 0.059 & 0.027 & -0.155 & 0.104 & 0.140 \\
\multirow{2}{*}{ Una/k } & $\mathrm{H}$ & -0.127 & 0.055 & 0.021 & -0.064 & 0.045 & 0.156 & -0.159 & 0.071 & 0.027 \\
& $\mathrm{NH}$ & -0.035 & 0.146 & 0.812 & -0.033 & 0.134 & 0.805 & -0.205 & 0.201 & 0.309 \\
\hline
\end{tabular}

Table 4. Multiple linear regression analysis for the association of FEna, FEk, and Una/k (independent variable) with FEur (dependent variable) in hypertension and non-hypertension group. model 1 adjusted for sex, age and BMI; model 2 plus eGFR and SUA; model 3 plus FBG, 24-hUg, TG, CRP, LDL, and TCO2. Beta coefficients refer to how many deviations a dependent variable will change per deviation increase in the predictor variable. SE refers to standard error. H: hypertension group; NH: non-hypertension group. Abbreviations: FEur, fractional excretion of uric acid; FEna, fractional excretion of sodium; FEk, fractional excretion of potassium; Una/k: urinary sodium/potassium ratio

\begin{tabular}{lcccccccccc}
\hline \multirow{2}{*}{ Parameter } & & \multicolumn{3}{c}{ Model 1 } & \multicolumn{3}{c}{ Model 2 } & \multicolumn{3}{c}{ Model 3 } \\
& & $\mathrm{B}$ & $\mathrm{SE}$ & $\mathrm{P}$ & $\mathrm{B}$ & $\mathrm{SE}$ & $\mathrm{P}$ & $\mathrm{B}$ & SE & $\mathrm{P}$ \\
\hline \multirow{2}{*}{ FEna (\%) } & $\mathrm{H}$ & 2.251 & 0.125 & $<0.001$ & 2.103 & 0.139 & $<0.001$ & 1.641 & 1.90 & $<0.001$ \\
& $\mathrm{NH}$ & 2.438 & 0.474 & $<0.001$ & 1.862 & 0.563 & 0.001 & 0.713 & 0.961 & 0.460 \\
\multirow{2}{*}{ FEk (\%) } & $\mathrm{H}$ & 0.533 & 0.030 & $<0.001$ & 0.545 & 0.034 & $<0.001$ & 0.576 & 0.051 & $<0.001$ \\
& $\mathrm{NH}$ & 0.327 & 0.093 & 0.001 & 0.127 & 0.115 & 0.273 & 0.060 & 0.197 & 0.750 \\
\multirow{2}{*}{ Una/k } & $\mathrm{H}$ & 1.169 & 0.200 & $<0.001$ & 0.727 & 0.187 & $<0.001$ & 0.578 & 0.227 & 0.012 \\
& $\mathrm{NH}$ & 0.766 & 0.266 & 0.004 & 0.481 & 0.254 & 0.06 & 0.118 & 0.381 & 0.757 \\
\hline
\end{tabular}

As for hypertension population, In HG1 group (eGFR $<33.9 \mathrm{ml} / \mathrm{min} / 1.73 \mathrm{~m}^{2}$ ), Spearman correlation analysis showed that both FEna and FEk was positively associated with FEur ( $\mathrm{r}=0.629, P<0.001 ; \mathrm{r}=0.655, P<0.001$, respectively). Multiple linear regression analysis showed that the association of FEna and FEk with FEur was still statistically significant (both $P<0.001$; Table 5). In HG2 group (33.9 $\leq \mathrm{eGFR}<66.6 \mathrm{ml} / \mathrm{min} / 1.73 \mathrm{~m}^{2}$ ), Spearman correlation analysis showed that both FEna and FEk was positively associated with FEur $(\mathrm{r}=0.268$, $P=0.014 ; \mathrm{r}=0.302, P=0.005$, respectively). Multiple linear regression analysis showed that the association of FEna and FEk with FEur disappeared (both $P>0.05$; Table 5). In HG3 group (eGFR $\geq 66.6 \mathrm{ml} / \mathrm{min} / 1.73 \mathrm{~m}^{2}$ ), Spearman correlation analysis showed that FEna was positively associated with FEur $(\mathrm{r}=0.534, P<0.001)$, and FEk was not related to FEur $(\mathrm{r}=0.169, P=0.133)$. Multiple linear regression analysis showed that the association of FEna with FEur disappeared $(P>0.05$, Table 5).

As to non-hypertension population, In NHG1 group (eGFR $<68.4 \mathrm{ml} / \mathrm{min} / 1.73 \mathrm{~m}^{2}$ ), Spearman correlation analysis showed that FEna and FEk was positively associated with FEur ( $\mathrm{r}=0.674, P<0.001 ; \mathrm{r}=0.421, P=0.001$, respectively). Multiple linear regression analysis showed that the association of FEna and FEk with FEur disappeared (both $P>0.05$; Table 6). In NHG2 group ( $\left.68.4 \leq \mathrm{eGFR}<98.5 \mathrm{ml} / \mathrm{min} / 1.73 \mathrm{~m}^{2}\right)$, Spearman correlation analysis showed that FEna was positively associated with FEur ( $\mathrm{r}=0.372, P=0.006)$, and FEk was not related to FEur $(\mathrm{r}=0.159, P=0.250)$. Multiple linear regression analysis showed that the association of FEna with FEur disappeared ( $P>0.05$; Table 6). In NHG3 group (eGFR $\geq 98.5 \mathrm{ml} / \mathrm{min} / 1.73 \mathrm{~m}^{2}$ ), Spearman correlation analysis showed that both FEna and FEk was positively associated with FEur ( $\mathrm{r}=0.415, P=0.001 ; \mathrm{r}=0.396, P=0.003$, respectively). Multiple linear regression analysis showed that the association of FEna and FEk with FEur disappeared (both $P>0.001$; Table 6). 


\section{Kidney Blood Pressure Research}

Differences in urinary uric acid excretion according to tertiles of urinary sodium and potassium in hypertension group

Compared with the first tertile of FEna group, levels of 24-h Uur and Cur in the highest tertile of FEna group were lower (both $P<0.001$; Fig.1A and 1B), and levels of FEur in the second tertile and highest tertile of FEna groups were higher (both $P<0.001$; Fig.1C). And

Table 5. Multiple linear regression analysis for the association of FEna, FEk, and Una/k with FEur in three groups by tertiles of eGFR in hypertension population. model 1 adjusted for sex, age and BMI; model 2 plus eGFR and SUA; model 3 plus FBG, 24-hUg, TG, CRP, LDL, and TCO2. Beta coefficients refer to how many deviations a dependent variable will change per deviation increase in the predictor variable. SE refers to standard error. HG1 group: eGFR<33.9ml/min/1.73m²; HG2 group: $33.9 \leq \mathrm{eGFR}<66.6 \mathrm{ml} / \mathrm{min} / 1.73 \mathrm{~m}^{2}$; HG3 group: eGFR $\geqslant 66.6 \mathrm{ml} / \mathrm{min} / 1.73 \mathrm{~m}^{2}$. Abbreviations: FEur, fractional excretion of uric acid; FEna, fractional excretion of sodium; FEk, fractional excretion of potassium

\begin{tabular}{|c|c|c|c|c|c|c|c|c|c|c|}
\hline \multirow{2}{*}{\multicolumn{2}{|c|}{ Parameter }} & \multicolumn{3}{|c|}{ Model 1} & \multicolumn{3}{|c|}{ Model 2} & \multicolumn{3}{|c|}{ Model 3} \\
\hline & & B & $\mathrm{SE}$ & P & B & $\mathrm{SE}$ & P & B & $\mathrm{SE}$ & $\mathrm{P}$ \\
\hline \multirow{2}{*}{ HG1 } & FEna & 1.973 & 0.184 & $<0.001$ & 1.368 & 0.226 & $<0.001$ & 1.195 & 0.308 & $<0.001$ \\
\hline & FEk & 0.510 & 0.051 & $<0.001$ & 0.361 & 0.057 & $<0.001$ & 0.504 & 0.085 & $<0.001$ \\
\hline \multirow{2}{*}{ HG2 } & FEna & 4.615 & 0.638 & $<0.001$ & 6.190 & 0.610 & $<0.001$ & 2.545 & 4.677 & 0.592 \\
\hline & FEk & 0.699 & 0.102 & $<0.001$ & 0.890 & 0.100 & $<0.001$ & 0.063 & 0.362 & 0.863 \\
\hline HG3 & FEna & 1.627 & 0.929 & 0.084 & 1.220 & 0.706 & 0.088 & 1.419 & 0.914 & 0.129 \\
\hline
\end{tabular}

Table 6. Multiple linear regression analysis for the association of FEna, FEk, and Una/k with FEur in three groups by tertiles of eGFR in non-hypertension population. model 1 adjusted for sex, age and BMI; model 2 plus eGFR and SUA; model 3 plus FBG, 24-hUg, TG, CRP, LDL, and TCO2. Beta coefficients refer to how many deviations a dependent variable will change per deviation increase in the predictor variable. SE refers to standard error. NHG1 group: eGFR $<68.4 \mathrm{ml} / \mathrm{min} / 1.73 \mathrm{~m}^{2}$; NHG2 group: $68.4 \leq \mathrm{eGFR}<98.5 \mathrm{ml} / \mathrm{min} / 1.73 \mathrm{~m}^{2}$; NHG3 group: eGFR $\geq 98.5 \mathrm{ml} / \mathrm{min} / 1.73 \mathrm{~m}^{2}$ Abbreviations: FEur, fractional excretion of uric acid; FEna, fractional excretion of sodium; FEk, fractional excretion of potassium

\begin{tabular}{lcccccccccc}
\hline \multirow{2}{*}{ Parameter } & & \multicolumn{3}{c}{ Model 1 } & \multicolumn{3}{c}{ Model 2 } & \multicolumn{3}{c}{ Model 3 } \\
& & B & SE & \multicolumn{1}{c}{ P } & B & SE & P & B & SE & P \\
& FEna & 1.751 & 0.963 & 0.075 & 0.733 & 1.343 & 0.588 & 0.126 & 2.579 & 0.961 \\
\multirow{2}{*}{ NHG1 } & FEk & 0.142 & 0.197 & 0.476 & 0.447 & 0.279 & 0.116 & 0.496 & 0.540 & 0.369 \\
\multirow{2}{*}{ NHG3 } & FEna & 2.111 & 0.991 & 0.038 & 1.846 & 0.736 & 0.016 & 1.182 & 1.139 & 0.312 \\
& FEna & 7.794 & 1.000 & $<0.001$ & 7.493 & 0.878 & $<0.001$ & 1.134 & 1.885 & 0.554 \\
& FEk & 0.643 & 0.142 & $<0.001$ & 0.640 & 0.126 & $<0.001$ & 0.244 & 0.135 & 0.084 \\
\hline
\end{tabular}

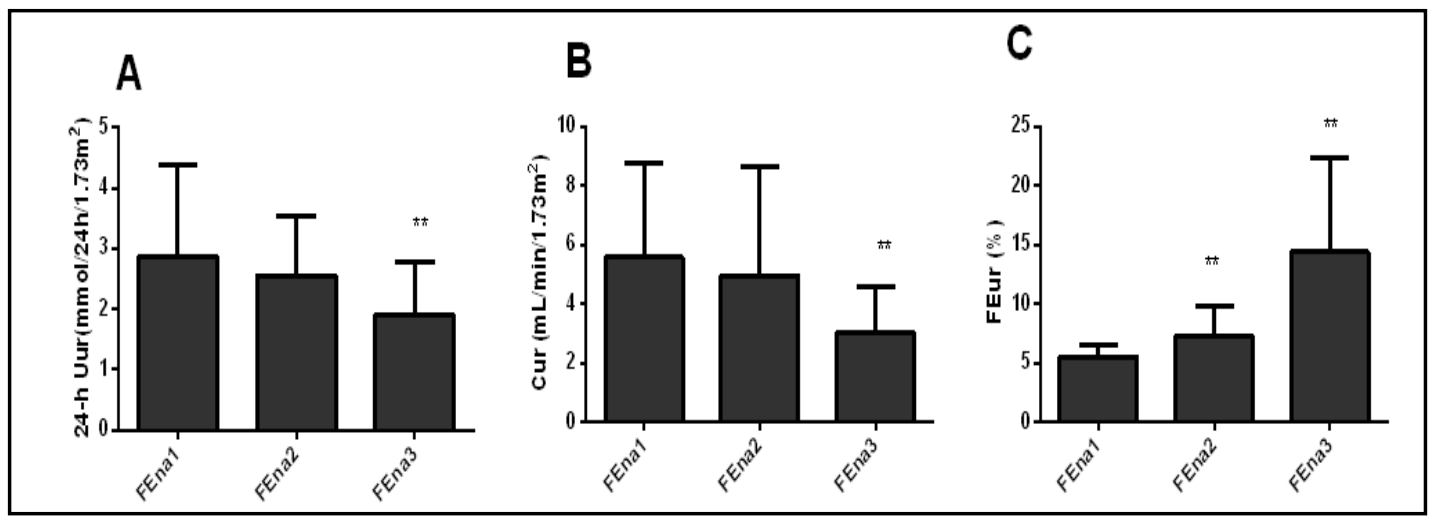

Fig. 1. Differences in 24-h Uur, Cur and FEur according to tertiles of FEna in hypertension group. Levels of 24-h Uur (A), Cur (B) and FEur (C) were compared according to tertiles of FEna using one-way ANOVA. Compared with the first tertile of FEna group, levels of 24-h Uur and Cur in the highest tertile of FEna group were lower (A and B, respectively), and levels of FEur in the second tertile and highest tertile of FEna groups were higher (C). FEna1: FEna $<0.92 \%$; FEna2: $0.92 \% \leq$ FEna $\leq 1.88 \%$; FEna3: FEna $>1.88 \%$ ). ${ }^{*} \mathrm{P}<0.05$, ${ }^{* *}$ $\mathrm{P}<0.01$ vs. Fna1. Abbreviations: 24 -h Uur, 24-hour urinary uric acid excretion; Cur, uric acid clearance rate; FEur, fractional excretion of uric acid; FEna, fractional excretion of sodium; ANOVA, analysis of variance. 


\section{Kidney Blood Pressure Research}

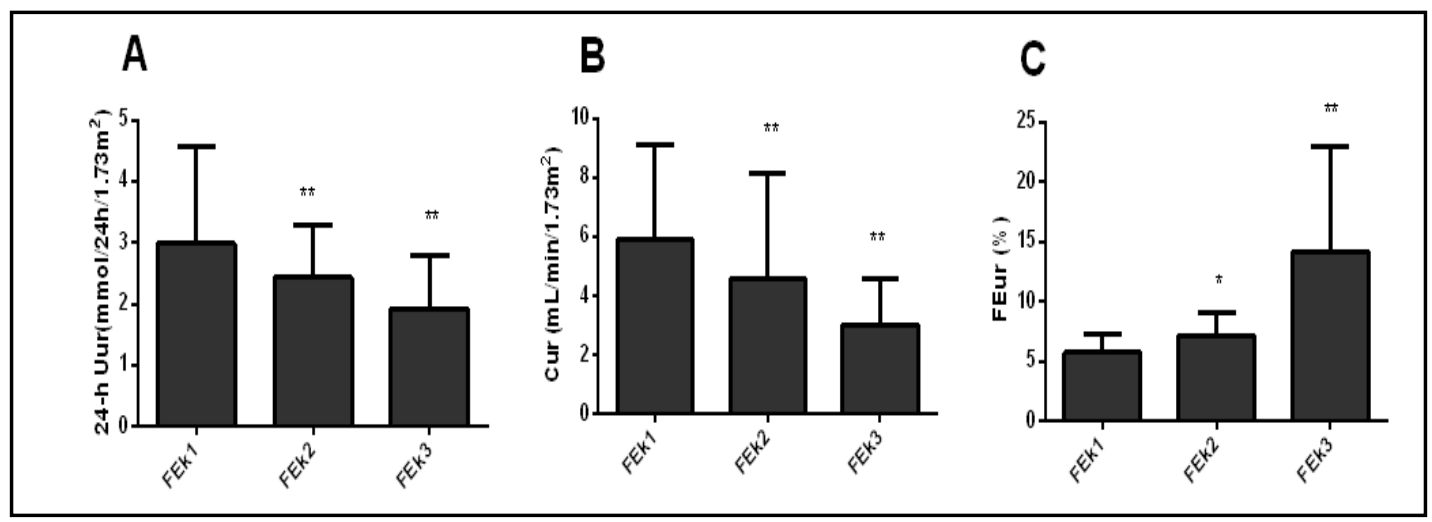

Fig. 2. Differences in 24-h Uur, Cur and FEur according to tertiles of FEk in hypertension group. Levels of 24-h Uur (A), Cur (B) and FEur (C) were compared according to tertiles of FEk using one-way ANOVA. Participants in the second tertile and highest tertile of FEk groups had lower 24-h Uur and Cur and higher FEur than those in the first tertile of FEk group. FEk1: FEk<8.36\%; FEk2: 8.36\% $\leq$ FEk $\leq 15.17 \%$; FEk3: FEk $>15.17 \%$. ${ }^{*} \mathrm{P}<0.05,{ }^{* *} \mathrm{P}<0.01$ vs. FEk1. Abbreviations: 24 -h Uur, 24-hour urinary uric acid excretion; Cur, uric acid clearance rate; FEur, fractional excretion of uric acid; FEk, fractional excretion of potassium; ANOVA, analysis of variance.
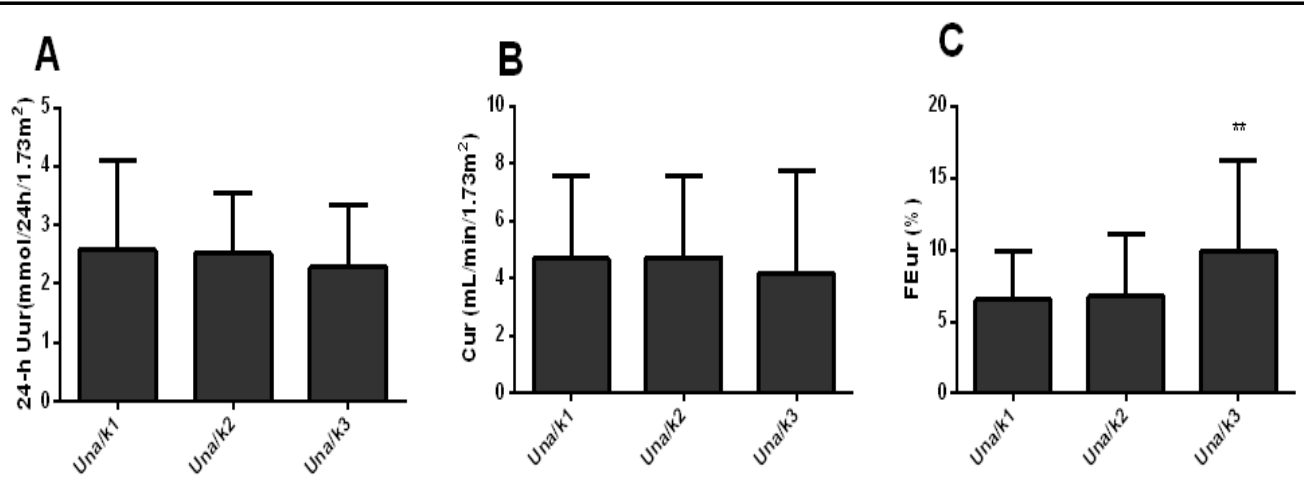

Fig. 3. Differences in 24-h Uur, Cur and FEur according to tertiles of Una/k in hypertension group. Levels of 24-h Uur (A), Cur (B) and FEur (C) were compared according to tertiles of Una/k using one-way ANOVA. FEur in the highest tertile of Una/k group was higher than that in the first tertile of Una group. But there was no statistical difference for levels of 24-h Uur and Cur in different tertiles of Una/k groups. Una/k1: Una/ k<3.86; Una/k2: $3.86 \leq \mathrm{Una} / \mathrm{k} \leq 5.40$; Una/k3: Una/k>5.40. ${ }^{*} \mathrm{P}<0.05$, ${ }^{* *} \mathrm{P}<0.01$ vs. Una/k1. Abbreviations: 24-h Uur, 24-hour urinary uric acid excretion; Cur, uric acid clearance rate; FEur, fractional excretion of uric acid; Una/k: urinary sodium/potassium ratio. ANOVA, analysis of variance.

participants in the second tertile and highest tertile of FEk group had lower 24-h Uur and Cur and higher FEur than those in the first tertile of FEk group (all $P<0.05$; Fig. 2). FEur in the highest tertile of Una/k group was higher than that in the first tertile of Una/k group $(P<0.001$; Fig.3C). But there was no statistical difference for levels of 24-h Uur and Cur in different tertiles of Una/k groups $(P>0.05$; Fig. $3 A$ and $B)$.

\section{Discussion}

To our knowledge, this study is the first to investigate the association of urinary sodium and potassium with renal handling of uric acid among patients with CKD. After adjusting for many potential confounders, we found that in hypertension group, fractional excretion of sodium (FEna) was negatively correlated with 24 -hUur and Cur, and positively correlated 


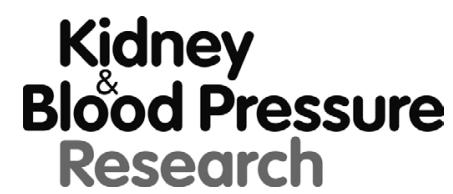

Kidney Blood Press Res 2018;43:1310-1321

\begin{tabular}{|l|l|}
\hline DOI: $10.1159 / 000492590$ & (c) 2018 The Author(s). Published by S. Karger AG, Basel \\
\hline
\end{tabular}

Fengqin et al:: Association of Urinary $\mathrm{Na}^{+}$and $\mathrm{K}^{+}$with Uric Acid

with FEur. Additionally, FEk was positively correlated with FEur, but not related to 24-hUur and Cur. And Una/k was negatively related to 24-h Uur and Cur, and positively related to FEur. In non-hypertension group, FEna was negatively correlated with 24-hUur, but not related to Cur and FEur. Moreover, both FEk and Una/k were not related to 24-h Uur, Cur and FEur. Furthermore, we investigated how the association of FEna and FEk with FEur tracks with changes in eGFR. The result showed that in hypertension population, FEna and FEk was still positively related to FEur in the lowest tertile of eGFR groups, but not related in the second and highest tertile of eGFR groups. In non-hypertension population, FEna and FEk was still not associated with FEur in all tertile of eGFR groups, after adjusting for many potential confounders.

Epidemiological studies have shown an association between high sodium and low potassium intake or a high urinary sodium/potassium ratio and high BP or cardiovascular disease $[8,15-17]$, which may play an important role in the pathogenesis of hypertension independent of cardiovascular risk factors. In this study, by analyzing CKD patients with and without hypertension, we observed that Una/k was negatively associated with 24-h Uur and Cur, and positively associated with FEur in hypertensive patients. However, these associations disappeared in non-hypertensive patients, after adjusting for many potential confounders. It suggested that urinary sodium and potassium excretion was closely related to renal handling of uric acid in hypertensive patients, which may be one of the mechanisms of the relationship between hyperuricemia and hyperuricemia. It is expected to help reduce the risk of hyperuricemia through a low sodium and high potassium diet.

The current relationship of urinary sodium with renal handling of uric acid is not clear. Recently, Perez-Ruiz found that renal clearance of uric acid was positively with fractional excretion of sodium, conducted in a untreated and non-diabetic gout patients [18]. In contrast, we observed that FEna was negatively correlated with Cur and 24-h Uur, and both FEna and FEk was positively correlated with FEur in hypertensive group with low eGFR. This seemed to reflect the fact that FEna and FEur increased adaptively, 24-hUur and Cur decreased with the decline of renal function in our CKD patients, according to our data and previous studies $[19,20]$. However, FEna and FEk was not related to FEur in hypertension population with early and medium-term decline in renal function (eGFR $\geq 33.9 \mathrm{ml} / \mathrm{min} / 1.73 \mathrm{~m}^{2}$ ) and nonhypertension population in our study. One potential explanation was that the mechanism of hypertension and hyperuricemia in CKD subjects with severely impaired GFR may relate to the direct effects of low GFR to impair sodium, potassium and uric acid handling [3, 21, 22]; as a consequence, this may be the main mechanism responsible for association FEna and FEk with FEur in hypertension patients as GFR decreased obviously, which may explain the whole relationship. Gout patients in the study above had normal renal function, which made a difference from ours. Indeed, early study also indicated that high serum uric acid levels were independently associated with increased proximal tubular sodium reabsorption in men workers. Concurrently, it also suggested that altered tubular sodium handling and uric acid metabolism were consistent with hyperinsulinemia, and insulin resistance could be the possible pathophysiological link [23]. Furthermore, subsequent researches observed a reduction in renal handling of both uric acid and sodium in healthy humans with acute physiological hyperinsulinemia and in hypertension [13]. According to previous studies, hyperinsulinemia could activate uric acid transporters and increase reabsorption of sodium and potassium in hypertensive subjects [24]. Moreover, hyperinsulinemia increases intracellular $\mathrm{pH}$ and activates the renal tubular $\mathrm{Na}^{+}-\mathrm{H}^{+}$exchange, followed by reabsorption of anions and urate salts $[25,26]$. However, in the present study, insulin resistance was not incorporated into the analysis due to absence of laboratory indicators reflecting insulin resistance, and further clinical and experiment studies should be done.

It is well known that the mechanism of renal tubular reabsorption and secretion of uric acid is complicated, mediated by various uric acid transporters at the apical and basolateral membrane of proximal epithelial tubular cells [27]. Uric acid reabsorption overall is supposed to be a tertiary active transport process based on sodium reabsorption, whereby the 
basolateral $\mathrm{Na}^{+}-\mathrm{K}^{+}$-ATPase generates a $\mathrm{Na}^{+}$gradient (primary active transport) that drives a number of apical urate transporters [6]. Up to date, urate transporter 1(URAT1) and glucose transporter-like protein-9 (GLUT9) are the best characterized urate transporters, mainly responsible for renal tubular reabsorption [27]. URAT1 (SLC22A12) is a major transporter of the organic anions transporter (OAT) family [28], and there is evidence for indirect coupling of sodium [29]. Since the molecular cloning of URAT1 (SLC22A12), several membrane proteins relevant to the transport of urate have been identified, such as SMCT1(SLC5A8) and SMCT2(SLC5A12). These proteins are sodium-dependent monocarboxylic acid transporters located in the proximal tubule that transport lactate and nicotinate into the proximal tubule cell $[30,31]$. Given these data, urinary sodium may have an inherent relationship with the reabsorption of uric acid by renal tubules. Indeed, CKD patients often present low urinary excretion of sodium, which was not only the main pathogenic basis of hypertension [22, 32], but also may be one of the mechanisms that affect the level of serum uric acid.

Currently, no population-based studies have been done on the association between urinary potassium excretion and renal handling of uric acid. In our study, we documented that urinary potassium was correlated with urinary excretion of uric acid in CKD patients with hypertension, after adjusting for potential confounders. Animals studies on physiological mechanism of renal tubule excretion of uric acid can explain our finding. In the perfused snake tubule preparation, Dantzler observed that the presence of $\mathrm{K}^{+}$in the bath was necessary to attain appreciable transport of uric acid, suggesting that uric acid is specifically stimulated by $\mathrm{K}^{+}$in the snake [33]. On the other hand, Chonko found that $10^{-4} \mathrm{M}$ ouabain almost completely inhibited the secretory flux of uric acid in the rabbit [34]. And ouabain could inhibited the activity of $\mathrm{Na}^{+}-\mathrm{K}^{+}$-ATPase and transport of $\mathrm{K}^{+}[35]$, which suggested urinary potassium was associated with tubular uric acid transport. In addition, a study on mechanisms of uric acid transport were investigated in human renal brush-border membrane vesicles, and it observed that uric acid transport in brush border membrane vesicle (BBMV) of human kidney depended on membrane potential. When an inward-positive potential was induced by imposing a $\mathrm{K}^{+}$gradient in the presence of valinomycin, a stimulation of uric acid uptake was obtained [36], which may be also one of the evidences of correlation between potassium transport and uric acid transport in renal tubule.

Some potential limitations of the present study should be noted. First, the cross-sectional nature of the present study makes it hard to establish the causal relationship between urinary sodium and potassium excretion with renal handling of uric acid. Second, a single urine collection might have overestimated or underestimated the actual urinary excretion of uric acid, sodium and potassium in the CKD population. Finally, we did not explore the effects of specific etiology of CKD on uric acid excretion. Nonetheless, there are still several noteworthy strengths to our present study. Most importantly, strict exclusion criteria based on medical histories and routine laboratory findings and careful adjustments for possible confounders was applied. In addition, we used combined indexes of urinary sodium, potassium and uric acid for the analysis, which was more comprehensive and representative.

\section{Conclusion}

The present study reported that in CKD patients, urinary sodium and potassium excretion was closely correlated with renal handling of uric acid after adjusting for multiple confounders, which was pronounced in hypertensive patients. This phenomenon may be one of the mechanisms of the relationship between hyperuricemia and hyperuricemia. Further research is needed to confirm it. It is expected to help reduce the risk of hyperuricemia through a low sodium and high potassium diet. 


\section{Kidney \\ Blood Pressure Research}

\begin{tabular}{l}
\hline Kidney Blood Press Res 2018;43:1310-1321 \\
\begin{tabular}{l|l} 
DOI: 10.1159/000492590 & $\begin{array}{l}\text { @ 2018 The Author(s). Published by S. Karger AG, Basel } \\
\text { www.karger.com/kbr }\end{array}$ \\
\hline Published online: 10 August, 2018
\end{tabular} \\
\hline
\end{tabular}

Fengqin et al.: Association of Urinary $\mathrm{Na}^{+}$and $\mathrm{K}^{+}$with Uric Acid

\section{Acknowledgements}

This research was supported by Shanghai Municipal Commission of Health and Family Planning, Key developing disciplines (Grant number 2015ZB0501).

\section{Disclosure Statement}

All authors declared no competing interests.

\section{References}

1 Bellomo G: The relationship between uric acid, allopurinol, cardiovascular events, and kidney disease progression: A step forward. Am J Kidney Dis 2015;65:525-527.

2 Yan D, Tu Y, Jiang F, Wang J, Zhang R, Sun X, Wang T, Wang S, Bao Y, Hu C, Jia W: Uric Acid is independently associated with diabetic kidney disease: A cross-sectional study in a Chinese population. Plos One 2015;10:e129797.

- J Johnson RJ, Nakagawa T, Jalal D, Sanchez-Lozada LG, Kang DH, Ritz E: Uric acid and chronic kidney disease: Which is chasing which? Nephrol Dial Transplant 2013;28:2221-2228.

4 Nugent CA, Tyler FH: The renal excretion of uric acid in patients with gout and in nongouty subjects. J Clin Invest 1959;38:1890-1898.

5 Perez-Ruiz F, Calabozo M, Erauskin GG, Ruibal A, Herrero-Beites AM: Renal underexcretion of uric acid is present in patients with apparent high urinary uric acid output. Arthritis Rheum 2002;47:610-613.

-6 Bobulescu IA, Moe OW: Renal transport of uric acid: Evolving concepts and uncertainties. Adv Chronic Kidney Dis 2012;19:358-371.

7 Strazzullo P, D’Elia L, Kandala NB, Cappuccio FP: Salt intake, stroke, and cardiovascular disease: Metaanalysis of prospective studies. BMJ 2009;339:b4567.

8 Mente A, O’Donnell MJ, Rangarajan S, McQueen MJ, Poirier P, Wielgosz A, Morrison H, Li W, Wang X, Di C, Mony P, Devanath A, Rosengren A, Oguz A, Zatonska K, Yusufali AH, Lopez-Jaramillo P, Avezum A, Ismail N, Lanas F, et al.: Association of urinary sodium and potassium excretion with blood pressure. N Engl J Med 2014;371:601-611.

9 Judd E, Calhoun DA: Management of hypertension in CKD: Beyond the guidelines. Adv Chronic Kidney Dis 2015;22:116-122.

10 Mills KT, Chen J, Yang W, Appel LJ, Kusek JW, Alper A, Delafontaine P, Keane MG, Mohler E, Ojo A, Rahman M, Ricardo AC, Soliman EZ, Steigerwalt S, Townsend R, He J: Sodium excretion and the risk of cardiovascular disease in patients with chronic kidney disease. JAMA 2016;315:2200-2210.

11 Mallat SG, Al KS, Tanios BY, Jurjus A: Hyperuricemia, hypertension, and chronic kidney disease: An emerging association. Curr Hypertens Rep 2016;18:74.

$\$ 12$ Krishnan E: Interaction of inflammation, hyperuricemia, and the prevalence of hypertension among adults free of metabolic syndrome: NHANES 2009-2010. J Am Heart Assoc 2014;3:e157.

13 Ter Maaten JC, Voorburg A, Heine RJ, Ter Wee PM, Donker AJ, Gans RO: Renal handling of urate and sodium during acute physiological hyperinsulinaemia in healthy subjects. Clin Sci (Lond) 1997;92:51-58.

14 Stevens PE, Levin A: Evaluation and management of chronic kidney disease: Synopsis of the kidney disease: Improving global outcomes 2012 clinical practice guideline. Ann Intern Med 2013;158:825-830.

15 Hedayati SS, Minhajuddin AT, Ijaz A, Moe OW, Elsayed EF, Reilly RF, Huang CL: Association of urinary sodium/potassium ratio with blood pressure: Sex and racial differences. Clin J Am Soc Nephrol 2012;7:315-322.

16 Kwok TC, Chan TY, Woo J: Relationship of urinary sodium/potassium excretion and calcium intake to blood pressure and prevalence of hypertension among older Chinese vegetarians. Eur J Clin Nutr 2003;57:299304. 


\section{Kidney \\ Blood Pressure Research}

17 Adrogue HJ, Madias NE: Sodium and potassium in the pathogenesis of hypertension. N Engl J Med 2007;356:1966-1978.

18 Perez-Ruiz F, Aniel-Quiroga MA, Herrero-Beites AM, Chinchilla SP, Erauskin GG, Merriman T: Renal clearance of uric acid is linked to insulin resistance and lower excretion of sodium in gout patients. Rheumatol Int 2015;35:1519-1524.

19 Steele TH, Rieselbach RE: The contribution of residual nephrons within the chronically diseased kidney to urate homeostasis in man. Am J Med 1967;43:876-886.

-20 Sorensen LF: Gout secondary to chronic renal disease: Studies on urate metabolism. Ann Rheum Dis 1980;39:424-430.

-21 Suliman ME, Johnson RJ, Garcia-Lopez E, Qureshi AR, Molinaei H, Carrero JJ, Heimburger O, Barany P, Axelsson J, Lindholm B, Stenvinkel P: J-shaped mortality relationship for uric acid in CKD. Am J Kidney Dis 2006;48:761-771.

-22 Ihm CG: Hypertension in chronic glomerulonephritis. Electrolyte Blood Press 2015;13:41-45.

23 Cappuccio FP, Strazzullo P, Farinaro E, Trevisan M: Uric acid metabolism and tubular sodium handling. Results from a population-based study. JAMA 1993;270:354-359.

-24 Muscelli E, Natali A, Bianchi S, Bigazzi R, Galvan AQ Sironi AM, Frascerra S, Ciociaro D, Ferrannini E: Effect of insulin on renal sodium and uric acid handling in essential hypertension. Am J Hypertens 1996;9:746752.

25 Osgood K, Krakoff J, Thearle M: Serum uric acid predicts both current and future components of the metabolic syndrome. Metab Syndr Relat Disord 2013;11:157-162.

26 Zapolski T, Wacinski P, Kondracki B, Rychta E, Buraczynska MJ, Wysokinski A: Uric acid as a link between renal dysfunction and both pro-inflammatory and prothrombotic state in patients with metabolic syndrome and coronary artery disease. Kardiol Pol 2011;69:319-326.

-27 Hyndman D, Liu S, Miner JN: Urate handling in the human body. Curr Rheumatol Rep 2016;18:34.

-28 Enomoto A, Kimura H, Chairoungdua A, Shigeta Y, Jutabha P, Cha SH, Hosoyamada M, Takeda M, Sekine T, Igarashi T, Matsuo H, Kikuchi Y, Oda T, Ichida K, Hosoya T, Shimokata K, Niwa T, Kanai Y, Endou H: Molecular identification of a renal urate anion exchanger that regulates blood urate levels. Nature 2002;417:447-452.

29 Anzai N, Jutabha P, Amonpatumrat-Takahashi S, Sakurai H: Recent advances in renal urate transport: Characterization of candidate transporters indicated by genome-wide association studies. Clin Exp Nephrol 2012;16:89-95.

-30 Srinivas SR, Gopal E, Zhuang L, Itagaki S, Martin PM, Fei YJ, Ganapathy V, Prasad PD: Cloning and functional identification of slc5a12 as a sodium-coupled low-affinity transporter for monocarboxylates (SMCT2). Biochem J 2005;392:655-664.

31 Anzai N, Kanai Y, Endou H: New insights into renal transport of urate. Curr Opin Rheumatol 2007;19:151157.

-32 Ando K, Fujita T: Pathophysiology of salt sensitivity hypertension. Ann Med 2012;44:S119-S126.

33 Berndt WO, Beechwood EC: Influence of inorganic electrolytes and ouabain on uric acid transport. Am J Physiol 1965;208:642-648.

34 Chonko AM: Urate secretion in isolated rabbit renal tubules. Am J Physiol 1980;239:F545-F551.

-35 Dantzler WH: Effects of $\mathrm{K}, \mathrm{Na}$, and ouabain on urate and PAH uptake by snake and chicken kidney slices. Am J Physiol 1969;217:1510-1519.

-36 Roch-Ramel F, Werner D, Guisan B: Urate transport in brush-border membrane of human kidney. Am J Physiol 1994;266:F797-F805. 